\title{
Prenatal chromosomal microarray analysis of fetus with increased nuchal translucency
}

\author{
So Hyun Shim and Dong Hyun Cha* \\ Department of Obstetrics and Gynecology, CHA Gangnam Medical Center, CHA University, Seoul, Korea
}

\begin{abstract}
Nuchal translucency is an important indicator of an aneuploid fetus in prenatal diagnostics. Previously, only the presence of aneuploid could be confirmed by conventional karyotyping of fetuses with thick nuchal translucency. With the development of genetic diagnostic techniques, however, it has been reported that subtle variations not detectable by conventional karyotyping might occur in cases of pathologic clinical syndrome in euploid fetuses. One of the newer, high-resolution genetic methods in the prenatal setting is chromosomal microarray. The possible association between nuchal translucency thickness with normal karyotype and submicroscopic chromosomal abnormalities detectable by microarray has been studied. How and when to apply microarray in clinical practice, however, is still debated. This article reviews the current studies on the clinical application of microarray in cases of increased nuchal translucency with normal karyotype for prenatal diagnosis.
\end{abstract}

Key words: Nuchal translucency measurement, Microarray analysis, Comparative genomic hybridization, Prenatal diagnosis.

\section{Introduction}

As genetic technology has advanced dramatically over the past few decades and the understanding of pregnant women in prenatal care has increased, the importance of more specialized prenatal care has been emphasized in clinical practice. Chromosomal analysis is an important diagnostic procedure owing to its high correlation with clinical phenotype in prenatal diagnostics.

Conventional cytogenetic analysis by Giemsa banding (Gbanding) has been applied to identify numerical and structural chromosomal abnormalities by microscopy. However, it yields a lower band resolution ( $>4 \mathrm{Mb}$ ) and cannot effectively detect submicroscopic chromosomal anomalies. Thus, numerous microdeletion syndromes go undetected by conventional karyotyping. Microarrays, one of the novel high-resolution methods, which utilizes large-insert clones, single-nucleotide polymorphism (SNP) arrays or oligo arrays, can detect deletions or duplications not identifiable by routine chromosomal analysis [1]. Microarray-based comparative genomic hybridization (CGH), based on a principle similar to that of conventional metaphase $\mathrm{CGH}$, includes, with small amounts of fetal DNA, probes that can be short oligonucleotides or genomic fragments up to $1 \mathrm{Mb}$, which can identify copy-number variation (CNV) that can cause clinically significant syndromes. CNV is an umbrella term used to denote the gains and losses of DNA sequences of over $1 \mathrm{~kb}$ in length [2]. These submicroscopic changes are found in clinical syndromes involving subtelomeric chromosomal deletions that account for about $6 \%$ of idiopathic mental retardation with or without congenital anomalies [3].

Invasive prenatal diagnostic analysis of chromosomal abnormalities, unfortunately, cannot be performed on all pregnant women, and so prenatal screening tests that select for high-risk

Received: 2 June 2018, Revised: 11 September 2018, Accepted: 12 September 2018, Published: 31 December 2018

${ }^{*}$ Corresponding author: Dong Hyun Cha, M.D., Ph.D. (iD http://orcid.org/0000-0003-0722-1714

Department of Obstetrics and Gynecology, CHA Gangnam Medical Center, CHA University, 566 Nonhyeon-ro, Gangnam-gu, Seoul 06135, Korea.

Tel: +82-2-3468-3132, Fax: +82-2-3468-2616, E-mail: chadh001@chamc.co.kr

Conflict of interest: The authors declare that they do not have any conflicts of interest.

(C) This is an open-access article distributed under the terms of the Creative Commons Attribution Non-Commercial License (http://creativecommons.org/licenses/by-nc/4.0/) which permits unrestricted non-commercial use, distribution, and reproduction in any medium, provided the original work is properly cited.

(c) Copyright 2018 by the Korean Society of Medical Genetics and Genomics 
groups are given priority. One of the important prenatal screening markers is fetal nuchal translucency (NT) thickness, which is measured by ultrasound in the first trimester of pregnancy. Fetal NT thickness over the 99th percentile ( $\geq 3.5 \mathrm{~mm}$ ) puts women at increased risk for aneuploidy [4-6]. So, fetuses with increased NT usually undergo conventional karyotyping with chorionic villi sampling to screen for aneuploidy. Fetuses with normal karyotype have been routinely followed up on as scheduled while any structural defects on ultrasound are investigated until delivery. However, it had been reported that such fetuses also are associated with increased risk of major fetal deficiencies, including major cardiac defects, various genetic syndromes and later fetal or perinatal death [7-9]. Recent studies have examined possible associations between increased NT and submicroscopic chromosomal abnormalities [10], and the clinical utility of array $\mathrm{CGH}$ in prenatal testing for detection of microdeletion diseases that can cause abnormal phenotypes in karyotypes has been proven [11]. There are still many and conflicting opinions on how to apply array $\mathrm{CGH}$ in real clinical settings and whether it should replace the existing conventional cytogenetics [12]. Herein, we review the current literature on the clinical application of array CGH for increased NT with normal karyotype in prenatal diagnostics.

\section{Chromosomal Microarray Analysis vs. Karyotyp- ing}

Chromosomal abnormalities, which represent changes in the number or structure of chromosomes visible under microscopic for a number of clinical conditions called chromosomal disorders. The 24 types of chromosomes found in the human genome can be easily identified by their cytologic level using specific staining procedures. The most common of these was G-banding, which was developed in the early 1970s and was the first widely used whole-genome analysis tool for research and clinical diagnostics. However, the sensitivity of G-banding at this resolution might be lower in the region where the banding pattern is less specific. Since the human genome is composed of about 3 billion base pairs, conventional chromosomal analysis at the level of 500 to 600 bands will contain 5 to 6 million base pairs per band. Therefore, it is difficult to detect chromosomal anomalies of less than $5 \mathrm{Mb}$ by general chromosome testing. Microdeletion syndrome, which presents a characteristic phenotype due to microdeletion of less than $5 \mathrm{Mb}$ in a specific genome, is an important aspect of congenital genetic disease [13]. Therefore, in order to diagnose microdeletion syndrome, polymerase chain reaction and fluorescence in situ hybridization (FISH) has been performed on causative genomes. The limitation of these methods, however, is that only the targeted region of the genome is detectable; as such, abnormalities in other genome regions cannot be identified. Recent advances in genomics have led to the development of chromosome microarrays capable of detecting ultrastructural microscopic genomic variations at a time, which methods currently are undergoing routine test in domestic and international clinical genetic laboratories. Two of the newer genetic methods in the prenatal setting are chromosomal microarray and whole-exome sequencing.

Chromosomal microarray analysis (CMA) is a means of measuring gains and losses of DNA throughout the human genome. CMA has been used initially to identify chromosomes and genomic abnormalities in children with unexplained developmental delays, intellectual disabilities, or congenital defects reflecting tiny pathogenic genomic alterations that cannot be detected with conventional G-banding. Based on the greatly increased yield that it affords, CMA has grown to replace G-banding as the front-line prenatal test for specific patient populations.

\section{Types of Chromosomal Microarray Analysis}

The types of CMA are classified into bacterial artificial chromosome (BAC) arrays, CGH arrays, and SNP arrays. The BAC array is the first CMA platform to use the BAC clone derived from the human genome project. Since then, the CGH and SNP arrays have been developed and widely employed worldwide. The BAC and $\mathrm{CGH}$ arrays are based on relative analyses of the genomic quantities of the test and reference genomes, and the SNP arrays is based on the distinction between the two SNP alleles at specific locations in the genome.

CMAs are used to measure increases and decreases in the number of copies of a particular region of the genome by providing extensive information about the genetic CNV. Depending on the density of the DNA probes installed on the chip, the results of both breakpoints, resolution and size of the predicted mutations are determined; thus, the performance of the assay depends on how precisely and at how high a density the probes are installed. Since the state of the mutation, in the form of gains or losses, is calculated based on the relative frequency with respect to the probe at the corresponding position, structural variations such as chromosome balance translocation or the inverse cannot be found, which is the limitation of CMA testing. 


\section{Chromosomal Microarray Analysis in Prenatal Diagnosis}

Currently, CMA is successfully applied for prenatal diagnosis of fetal submicroscopic chromosomal abnormalities using the chorionic villus or amniotic fluid of the fetus, and it is used in clinical practice outside South Korea. Prenatal CMA is expected to play a major role in the diagnosis of fetal chromosomal abnormalities by providing various information on fetal CNV. Prenatal CMA can detect minute chromosomal abnormalities, which makes possible the shortening of examination time by rendering the culturing of amniotic fluid or chorionic villus cells unnecessary. So, even in cases of stillbirth that are not well cultured, CMA testing is possible. Additionally, the test itself is automated and objective relative to microscopy-based conventional chromosomal testing. It is also possible to customize a platform on which detectors are concentrated on specific areas of the genome, such as dense regions, thereby enhancing the accuracy of the test. Moreover, the results can be more efficiently analyzed using vast amounts of data already in the database. However, CMA testing has disadvantages, in that with it, finding structural variation such as translocation or inversion, or mosaicism existing at a low rate of $20 \%$ or less, is impossible; also it is relatively expensive. Furthermore, since benign CNV or variation of unknown clinical significance (VOUS) might be detected, definitive guidelines for interpretation of results are essential. VOUS is still a serious challenge for genetic counseling experts. Indeed, it can lead to unnecessary maternal anxiety or even, due to uncertain prognoses, decisions to terminate pregnancy. Reducing its rate remains a significant problem to be resolved. Parental analysis is one useful strategy in this regard. Constant follow up of cases with VOUS to establish a wide-range database would be helpful for the purposes of prenatal consultation.

\section{Fetuses with Increased Nuchal Translucency and Normal Karyotype}

In the first trimester of pregnancy, subcutaneous collection of fluid in the fetal neck is visualized by ultrasound as NT. The measurement usually made between 11 and 14 week, and best view is obtained at 11 to 12 weeks. Generally NT thickness increases with fetal crown-rump length (CRL). NT thickness is between 1.2 and $2.1 \mathrm{~mm}$ at a CRL of $45 \mathrm{~mm}$ and is between 1.9 and 2.7 $\mathrm{mm}$ at a CRL of $84 \mathrm{~mm}$ (median and 95th percentile values). The 99th percentile regards as $3.5 \mathrm{~mm}$ [14].

First-trimester screening using a combination of age and
NT, pregnancy-associated plasma protein-A and b-human chorionic gonadotropin has been shown to be an effective way to screen for aneuploidy, demonstrating a detection rate of $90 \%$ at a false-positive rate of 5\% [15]. However, in fetuses with increased NT but normal karyotyping, nuchal thickening is clinically relevant due to its association with increased adverse perinatal outcomes caused by a variety of fetal malformations, dysplasias, deformations, dysruptions, and genetic syndromes [16-24]. In the combined data from 2 studies on a total of 4,540 chromosomally normal fetuses with increased NT but no obvious fetal defects on sonography, the prevalence of miscarriage or fetal death increased from 1.3\% in those with NT between the 95th and 99th percentiles (>6.5 mm) to about 20\% [22,23]. Another study of 6,650 pregnancies reported that in chromosomally normal fetuses, the prevalence of miscarriage or fetal death was $1.3 \%$ in those with NT below the 95th percentile, 1.2\% for NT between the 95th and 99th percentiles, and 12.3\% for NT above the 99th percentile [24]. Most of the later-stillborn fetuses in that study showed a tendency to progress to hydrops as the thickness of NT became aggravated.

Some studies have reported on the long-term follow-up of chromosomally and anatomically normal fetuses with increased NT. The prevalence of developmental delay was $2 \%$ in the combined total of 101 infants based on questionnaires to the parents [25-27]. On a combined total of 207 infants that had increased NT in fetal life, clinical examination demonstrated developmental delay in $3.9 \%$ of cases $[20,28-30]$. It is unclear whether these clinical phenotypes appearing in euploid fetuses were secondary consequences of other chromosomal abnormalities that could not be detected by conventional karyotyping methods, because not all of the fetuses with adverse outcomes were subjected to chromosomal analysis, and there are few studies with long-term follow up, even in cases of live birth.

As array CGH enables comprehensive high-resolution screening across the genome as well as mapping of DNA sequences not reachable by conventional karyotyping or FISH, the correlation of clinical consequences with microdeletion has to be established once the presence of aneuploidy is ruled out. The prevalence of numerous genetic disorders when NT is over 3.5 $\mathrm{mm}$ or over the 99th percentile with normal karyotype has been reported previously, but no definite association between increased NT and presence of genetic disorders was pointed out due to low incidence of some of the syndromes [31]. More than 50 genetic conditions have been identified as being in association with increased NT. The most commonly found disorders are DiGeorge syndrome (22q11.2 deletion), cat eye syndrome 
(22q11.2 duplication), Prader-Willi/Angelman syndrome (15q11q13 deletion), Williams syndrome (7q11.23 deletion), $1 q 21.1$ deletion/duplication syndrome, congenital adrenal hyperplasia, Noonan syndrome, Smith-Lemli-Opitz syndrome, fetal akinesia deformation sequence, multiple pterygium syndrome, Fanconi pancytopenia syndrome, campomelic dysplasia, and VATERL syndrome association [19-23,32] .

One recent meta-analysis of 18 studies reported pathogenic CNVs in 104 of 1,139 fetuses with multiple defects (9.1\%; 95\% confidence interval $[\mathrm{Cl}], 7.5-10.8)$ and in 125 of 2,220 fetuses with one anatomical system defect (5.6\%; 95\% Cl, 4.7-6.6) [33]. Among those cases, a larger number of submicroscopic chromosomal abnormalities were detected when more fetal structural defects were found. However, some studies have not shown any significant increase in euploid but anatomically anomalousy groups. Schou et al. [34] investigated 100 euploid fetuses with increased NT, and reported that none had pathogenic CNVs detectable by $\mathrm{CGH}$. However, three children were diagnosed with clinical syndromes postnatally; this might have been due to the fact that some other pathogenic CNVs were missed because the CGH platform used was able to detect only CNVs of $3 \mathrm{Mb}$ or more. Based on a study on 215 fetuses with increased NT, Huang et al. [35] reported that array CGH detected no cases of pathogenic CNVs in 199 euploid fetuses with no structural defects. In the remaining 16 cases with abnormal prenatal ultrasonography or abnormalities detected postnatally, CNVs classified as VOUS were later found. Yang et al. [36] reported that pathogenic CNVs were found in 26.9\% (7/26) of fetuses with both increased NT and anatomically anomaly on ultrasound at the second or third trimester, whereas for those fetuses with isolated increased NT, pathogenic CNVs were detected only in 6.7\% (13/194) of cases. Possible reasons for the variability among those results might have been differences in probe performance and in race-related characteristics. Inclusion of terminated fetuses with severe abnormality might also have resulted in higher rates of pathogenic CNVs. Due to this continuing uncertainty, how and when to apply this new technology is still under discussion. Some clinicians have insisted that detection of higher NT should prompt a detailed ultrasound examination for fetal abnormalities and, if at least one abnormalityi is detected, further, invasive-testingbased analysis should include array CGH. Some others have suggested that when invasive prenatal-testing procedures were required, array CGH should replace karyotyping [12]. Some investigators have detected over 30\% chromosomal (19.9\%) and submicroscopic chromosomal (9.1\%) abnormalities for fetuses with increased NT [36].

\section{Recommendations of Prenatal Microarray for Clinical Application}

Since the announcement of the use-of-CMA guidelines by the American College of Obstetricians and Gynecologists in 2009, the following 2016 amendments were announced. 1) Most genetic changes identified by CMA, typically not identified in standard karyotypes, are not associated with advanced maternal age; therefore, CMA testing can be considered for all women, regardless of age, who undergo prenatal testing. 2) Patients with a fetal structural abnormality on ultrasonography are recommended to undergo prenatal CMA in place of karyotyping. 3) Patients with a normal fetal structure on ultrasonography are advised to undergo karyotyping or prenatal CMA. 4) In the case of intrauterine fetal death or stillbirth, CMA testing using fetal tissue is recommended, as it can help to determine the causes of fetal death.

\section{Conclusion}

We reviewed the possible association between increased NT with normal karyotype and submicroscopic chromosomal abnormalities detected by array CGH. Array CGH has a higher resolution for detection and characterization of marker chromosomes and mosaicism within a shorter time than conventional and molecular cytogenetics. Although additional studies are needed to elucidate the relationship between increased NT and submicroscopic genetic syndrome, it is clear that CMA yields more genetic information than conventional karyotyping for the purposes of prenatal diagnosis. Also, the rate of CNV is increased significantly in case of furtrher combined other structural anomalies than simply isolated increased NT are found. In cases where invasive testing shows a normal chromosome genotype, it might be necessary to perform array CGH to eliminate the possibility of submicroscopic chromosomal abnormalities, especially when the fetus is found to have additional structural defects.

\section{References}

1. Coe BP, Ylstra B, Carvalho B, Meijer GA, Macaulay C, Lam WL. Resolving the resolution of array CGH. Genomics 2007;89:647-53.

2. lafrate $A$, Feuk L, Rivera MN, Listewnik ML, Donahoe PK, Qi Y, et al. Detection of large-scale variation in the human genome. Nat Genet 2004;36:949-51.

3. Knight SJ, Regan R, Nicod A, Horsley SW, Kearney L, Homfray T, et al. 
Subtle chromosomal rearrangements in children with unexplained mental retardation. Lancet 1999;354:1676-81.

4. Nicolaides $\mathrm{KH}$, Heath $\mathrm{V}$, Liao AW. The 11-14 week scan. Baillieres Best Pract Res Clin Obstet Gynaecol 2000;14:581-94.

5. Nicolaides $\mathrm{KH}$, Heath $\mathrm{V}$, Cicero $\mathrm{S}$. Increased fetal nuchal translucency at 11-14 weeks. Prenat Diagn 2002;22:308-15.

6. Cicero S, Sacchini C, Rembouskos G, Nicolaides KH. Sonographic markers of fetal aneuploidy--a review. Placenta 2003;24 Suppl B:S8898.

7. Atzei A, Gajewska K, Huggon IC, Allan L, Nicolaides KH. Relationship between nuchal translucency thickness and prevalence of major cardiac defects in fetuses with normal karyotype. Ultrasound Obstet Gynecol 2005;26:154-7.

8. Nicolaides $\mathrm{KH}$, Wegrzyn P. Fetal nuchal translucency thickness and risk for chromosomal defects. Ginekol Pol 2005;76:257-63.

9. Karagiannis G, Akolekar R, Sarquis R, Wright D, Nicolaides KH. Prediction of small-for-gestation neonates from biophysical and biochemical markers at 11-13 weeks. Fetal Diagn Ther 2011;29:148-54.

10. Leung TY, Vogel I, Lau TK, Chong W, Hyett JA, Petersen OB, et al. Identification of submicroscopic chromosomal aberrations in fetuses with increased nuchal translucency and apparently normal karyotype. Ultrasound Obstet Gynecol 2011;38:314-9.

11. Liao C, Li R, Fu F, Xie G, Zhang Y, Pan M, et al. Prenatal diagnosis of congenital heart defect by genome-wide high-resolution SNP array. Prenat Diagn 2014;34:858-63.

12. Kan AS, Lau ET, Tang WF, Chan SS, Ding SC, Chan KY, et al. Wholegenome array $\mathrm{CGH}$ evaluation for replacing prenatal karyotyping in Hong Kong. PLoS One 2014;9:e87988.

13. Schinzel A. Microdeletion syndromes, balanced translocations, and gene mapping. J Med Genet 1988;25:454-62.

14. Snijders RJ, Noble P, Sebire $N$, Souka A, Nicolaides KH; Fetal Medicine Foundation First Trimester Screening Group. UK multicentre project on assessment of risk of trisomy 21 by maternal age and fetal nuchal-translucency thickness at 10-14 weeks of gestation. Lancet 1998;352:343-6.

15. Mari G, Deter RL, Carpenter RL, Rahman F, Zimmerman R, Moise KJ Jr, et al; Collaborative Group for Doppler Assessment of the Blood Velocity in Anemic Fetuses. Noninvasive diagnosis by Doppler ultrasonography of fetal anemia due to maternal red-cell alloimmunization. N Engl J Med 2000;342:9-14.

16. Lam $Y H_{1}$ Tang MH, Lee CP, Tse HY. Nuchal translucency in fetuses affected by homozygous alpha-thalassemia-1 at 12-13 weeks of gestation. Ultrasound Obstet Gynecol 1999;13:238-40.

17. Zadeh $N$, Hudgins L, Norton ME. Nuchal translucency measurement in fetuses with spinal muscular atrophy. Prenat Diagn 2011;31:32730.
18. Barone $C$, Bianca S. Further evidence of no association between spinal muscular atrophy and increased nuchal translucency. Fetal Diagn Ther 2013;33:65-8.

19. Mangione R, Guyon F, Taine L, Wen ZQ, Roux D, Vergnaud A, et al. Pregnancy outcome and prognosis in fetuses with increased firsttrimester nuchal translucency. Fetal Diagn Ther 2001;16:360-3.

20. Senat MV, De Keersmaecker B, Audibert F, Montcharmont G, Frydman $R$, Ville Y. Pregnancy outcome in fetuses with increased nuchal translucency and normal karyotype. Prenat Diagn 2002;22:345-9.

21. Bilardo CM, Müller MA, Pajkrt E, Clur SA, van Zalen MM, Bijlsma EK. Increased nuchal translucency thickness and normal karyotype: time for parental reassurance. Ultrasound Obstet Gynecol 2007;30:11-8.

22. Mula $R$, Goncé $A$, Bennásar $M$, Arigita $M$, Meler $E_{1}$ Nadal $A$, et al. Increased nuchal translucency and normal karyotype: perinatal and pediatric outcomes at 2 years of age. Ultrasound Obstet Gynecol 2012; 39:34-41.

23. Pergament E, Alamillo C, Sak K, Fiddler M. Genetic assessment following increased nuchal translucency and normal karyotype. Prenat Diagn 2011;31:307-10.

24. Michailidis GD, Economides DL. Nuchal translucency measurement and pregnancy outcome in karyotypically normal fetuses. Ultrasound Obstet Gynecol 2001;17:102-5.

25. Maymon $R$, Jauniaux E, Cohen 0 , Dreazen E, Weinraub Z, Herman A. Pregnancy outcome and infant follow-up of fetuses with abnormally increased first trimester nuchal translucency. Hum Reprod 2000;15: 2023-7.

26. Van Vugt JM, Tinnemans BW, Van Zalen-Sprock RM. Outcome and early childhood follow-up of chromosomally normal fetuses with increased nuchal translucency at 10-14 weeks' gestation. Ultrasound Obstet Gynecol 1998;11:407-9.

27. Adekunle $\mathrm{O}$, Gopee $\mathrm{A}$, el-Sayed M, Thilaganathan B. Increased first trimester nuchal translucency: pregnancy and infant outcomes after routine screening for Down's syndrome in an unselected antenatal population. Br J Radiol 1999;72:457-60.

28. Hiippala A, Eronen M, Taipale $P$, Salonen R, Hiilesmaa V. Fetal nuchal translucency and normal chromosomes: a long-term follow-up study. Ultrasound Obstet Gynecol 2001;18:18-22.

29. Cheng CC, Bahado-Singh RO, Chen SC, Tsai MS. Pregnancy outcomes with increased nuchal translucency after routine Down syndrome screening. Int J Gynaecol Obstet 2004;84:5-9.

30. Brady AF, Pandya PP, Yuksel B, Greenough A, Patton MA, Nicolaides $\mathrm{KH}$. Outcome of chromosomally normal livebirths with increased fetal nuchal translucency at 10-14 weeks' gestation. J Med Genet 1998;35:222-4.

31. Souka AP, Von Kaisenberg CS, Hyett JA, Sonek JD, Nicolaides KH. Increased nuchal translucency with normal karyotype. Am J Obstet 
Gynecol 2005;192:1005-21.

32. Souka AP, Krampl E, Bakalis $S$, Heath V, Nicolaides KH. Outcome of pregnancy in chromosomally normal fetuses with increased nuchal translucency in the first trimester. Ultrasound Obstet Gynecol 2001; 18:9-17.

33. de Wit MC, Srebniak MI, Govaerts LC, Van Opstal D, Galjaard RJ, Go AT. Additional value of prenatal genomic array testing in fetuses with isolated structural ultrasound abnormalities and a normal karyotype: a systematic review of the literature. Ultrasound Obstet Gynecol 2014:43:139-46.

34. Schou KV, Kirchhoff M, Nygaard U, Jørgensen C, Sundberg K. In- creased nuchal translucency with normal karyotype: a follow-up study of 100 cases supplemented with CGH and MLPA analyses. UItrasound Obstet Gynecol 2009;34:618-22.

35. Huang J, Poon LC, Akolekar R, Choy KW, Leung TY, Nicolaides KH. Is high fetal nuchal translucency associated with submicroscopic chromosomal abnormalities on array CGH? Ultrasound Obstet Gynecol 2014;43:620-4.

36. Yang $X$, Li R, Fu F, Zhang Y, Li D, Liao C. Submicroscopic chromosomal abnormalities in fetuses with increased nuchal translucency and normal karyotype. J Matern Fetal Neonatal Med 2017;30:194-8. 